\title{
INCONSISTENCY LEMMAS IN ALGEBRAIC LOGIC
}

\author{
J.G. RAFTERY
}

\begin{abstract}
In this paper, the inconsistency lemmas of intuitionistic and classical propositional logic are formulated abstractly. We prove that, when a (finitary) deductive system $\vdash$ is algebraized by a variety $\mathrm{K}$, then $\vdash$ has an inconsistency lemma - in the abstract sense - iff every algebra in $\mathrm{K}$ has a dually pseudo-complemented join semilattice of compact congruences. In this case, the following are shown to be equivalent: (1) $\vdash$ has a classical inconsistency lemma; $(2) \vdash$ has a greatest compact theory and $\mathrm{K}$ is filtral - i.e., semisimple with EDPC; (3) the compact congruences of any algebra in $\mathrm{K}$ form a Boolean lattice; (4) the compact congruences of any $\boldsymbol{A} \in \mathrm{K}$ constitute a Boolean sublattice of the full congruence lattice of $\boldsymbol{A}$. These results extend to quasivarieties and relative congruences. Except for (2), they extend even to protoalgebraic logics, with deductive filters in the role of congruences. A protoalgebraic system with a classical inconsistency lemma always has a deductiondetachment theorem (DDT), while a system with a DDT and a greatest compact theory has an inconsistency lemma. The converses are false.
\end{abstract}

\section{INTRODUCTION}

In the axiomatic development of classical propositional logic, the inconsistency lemma emerges as a corollary of the deduction theorem, to be used in proving completeness. It states that, for any set $\Gamma \cup\{\alpha\}$ of formulas,

$$
\begin{aligned}
& \Gamma \cup\{\alpha\} \text { is inconsistent iff } \Gamma \vdash \neg \alpha ; \\
& \Gamma \cup\{\neg \alpha\} \text { is inconsistent iff } \Gamma \vdash \alpha .
\end{aligned}
$$

In intuitionistic propositional logic, (1) remains true, but (2) is false.

Here we shall be concerned with arbitrary deductive systems $\vdash$, but we always understand these to be finitary, i.e., whenever $\Gamma \vdash \alpha$, then $\Gamma^{\prime} \vdash \alpha$ for some finite $\Gamma^{\prime} \subseteq \Gamma$. The deduction-detachment theorem (or DDT) has a natural formulation in this general setting, where the concrete role of $\rightarrow$ is taken over by an unspecified set of binary formulas. It is well known that a system $\vdash$ has a DDT of this kind iff it is protoalgebraic and has a dually Brouwerian join semilattice of compact theories. In that case, the compact deductive filters of any algebra of the appropriate type are also dually Brouwerian, and $\vdash$ is filter-distributive with the filter extension property. For strongly algebraizable logics, the existence of a DDT amounts

Key words and phrases. Deductive system, inconsistency lemma, protoalgebraic logic, deduction-detachment theorem, algebraizable logic, pseudo-complement, filtral variety. 
to the equational definability of principal congruences in the corresponding algebras-briefly, EDPC. See Czelakowski $[12,15]$ and Blok et al. [3, 10].

As the theory of the DDT is rather satisfying, it is natural to ask whether a similarly abstract account of the inconsistency lemma can be given. This paper supplies such an account. Because (2) fails in intuitionistic logic, our basic definition generalizes (1). It has a specialization-called a classical inconsistency lemma - which abstracts the conjunction of (1) and (2).

We prove that a protoalgebraic deductive system has an inconsistency lemma in the general sense iff its join semilattice of compact theories is dually pseudo-complemented; it has a classical inconsistency lemma iff this semilattice is a Boolean lattice. In both cases, the characteristic properties of the compact theories are shared by the compact deductive filters of all algebras. In the classical case, the set of compact filters is closed under finite intersections. It follows on lattice-theoretic grounds that a protoalgebraic system with a classical inconsistency lemma has a DDT, while a system with a DDT and a greatest compact theory has an inconsistency lemma. The converses are false, even for strongly algebraizable logics.

We also show that, when a variety $\mathrm{K}$ algebraizes a deductive system $\vdash$ with a greatest compact theory, then $\vdash$ has a classical inconsistency lemma iff $\mathrm{K}$ is filtral-i.e., semisimple with EDPC. In that case, for all $\boldsymbol{A} \in \mathrm{K}$, the compact congruences of $\boldsymbol{A}$ form a Boolean sublattice of the congruence lattice of $\boldsymbol{A}$. Actually, these results are proved in slightly greater generality-for quasivarieties and relative congruences. Examples are discussed in Section 6.

Filtral classes were introduced by Magari [32, 33, 34]. They have been studied for a long time by many authors, e.g., [2, 5, 14, 21, 22, 23], but their connection with inconsistency lemmas seems to be new.

\section{Preliminaries}

From now on, $\vdash$ denotes a fixed but arbitrary (sentential) deductive system, i.e., a substitution-invariant finitary consequence relation over formulas in some algebraic language. Among other standard abbreviations, we signify ' $\Gamma \vdash \alpha$ for all $\alpha \in \Pi$ ' by $\Gamma \vdash \Pi$, and ' $\Gamma \vdash \Pi$ and $\Pi \vdash \Gamma$ ' by $\Gamma \dashv \vdash \Pi$.

We assume a familiarity with the basic theory of deductive systems and matrix semantics, cf. $[15,20,40]$. If $\langle\boldsymbol{A}, F\rangle$ is a matrix model of $\vdash$, then $F$ is called a $\vdash-$ filter of the algebra $\boldsymbol{A}$. Because the set of $\vdash$-filters of $\boldsymbol{A}$ is closed under arbitrary intersections, it becomes a complete lattice when ordered by set inclusion. This lattice is algebraic (as $\vdash$ is finitary), so its compact elements are just the finitely generated $\vdash$-filters of $\boldsymbol{A}$. In $\boldsymbol{A}$, the $\vdash-$ filter generated by a subset $Y$ is denoted as $\mathrm{Fg}_{\vdash}^{\boldsymbol{A}} Y$, while $F+{ }^{\boldsymbol{A}} G$ stands for the join of two $\vdash-$ filters $F$ and $G$. Thus, for instance, $F+{ }^{\boldsymbol{A}} \mathrm{Fg}_{\vdash}^{\boldsymbol{A}} Y=\mathrm{Fg}_{\vdash}^{\boldsymbol{A}}(F \cup Y)$. The universe of $\boldsymbol{A}$ is denoted as $A$.

Algebras are assumed to have the type of $\vdash$, unless we say otherwise. Recall that $\vdash$-theories are just $\vdash$-filters of the absolutely free algebra $\boldsymbol{F m}$ 
generated by the variables of $\vdash$, and substitutions are endomorphisms of Fm.

Since $\vdash$ has infinitely many variables, we may assume that two disjoint denumerable sequences of distinct variables,

$$
v_{1}, v_{2}, v_{3}, \ldots \text { and } x_{1}, x_{2}, x_{3}, \ldots,
$$

have been isolated for the rest of the discussion. We shall work mainly with the first sequence, using the second to avoid interpretational clashes. For each $n \in \mathbb{N}:=\{1,2,3, \ldots\}$, we define

$$
F m(n)=\left\{\beta \in F m \text { : the variables occurring in } \beta \text { are among } v_{1}, \ldots, v_{n}\right\} .
$$

If $\sigma \in F m(n)$ and $\boldsymbol{A}$ is an algebra, with $a_{1}, \ldots, a_{n} \in A$, then $\sigma^{\boldsymbol{A}}\left(a_{1}, \ldots, a_{n}\right)$ denotes $h(\sigma)$, where $h: \boldsymbol{F} \boldsymbol{m} \rightarrow \boldsymbol{A}$ is any homomorphism such that $h\left(v_{i}\right)=a_{i}$ for $i=1, \ldots, n$. If $\Xi \subseteq F m(n)$, then

$$
\Xi^{\boldsymbol{A}}\left(a_{1}, \ldots, a_{n}\right) \text { abbreviates }\left\{\xi^{\boldsymbol{A}}\left(a_{1}, \ldots, a_{n}\right): \xi \in \Xi\right\} \text {. }
$$

We omit the superscripts in $\sigma^{\boldsymbol{A}}, \Xi^{\boldsymbol{A}},+^{\boldsymbol{A}}$ and $\mathrm{Fg}_{\vdash}^{\boldsymbol{A}}$ when $\boldsymbol{A}$ is $\boldsymbol{F} \boldsymbol{m}$.

We need to recall two definitions and three results from abstract algebraic logic.

Definition 2.1. A congruence relation $\theta$ on an algebra $\boldsymbol{A}$ is said to be compatible with a subset $F$ of $A$ provided that $F$ is a union of $\theta$-classes, i.e., whenever $a \equiv_{\theta} b$ and $a \in F$, then $b \in F$.

Lemma 2.2. ([9]) Let $\langle\boldsymbol{A}, F\rangle$ and $\langle\boldsymbol{B}, G\rangle$ be matrix models of $\vdash$, and let $h: \boldsymbol{A} \rightarrow \boldsymbol{B}$ be a homomorphism of algebras. Then

(i) $h^{-1}[G]$ is a $\vdash$-filter of $\boldsymbol{A}$, and

(ii) if $h$ is surjective and ker $h$ is compatible with $F$, then $h[F]$ is a $\vdash$-filter of $\boldsymbol{B}$.

Here, as usual, ker $h:=\left\{\left\langle a, a^{\prime}\right\rangle \in A \times A: h(a)=h\left(a^{\prime}\right)\right\}$.

Definition 2.3. $([7,12,13]) \vdash$ is said to be protoalgebraic if there exists $\Lambda \subseteq F m(2)$ such that

$$
\begin{aligned}
& \vdash \Lambda\left(v_{1}, v_{1}\right) \text { and } \\
& \Lambda\left(v_{1}, v_{2}\right), v_{1} \vdash v_{2} .
\end{aligned}
$$

Note that $\Lambda$ can be chosen finite, because $\vdash$ is finitary.

Theorem 2.4. ([9, Thm. 7.6]) $\vdash$ is protoalgebraic iff the following is true whenever $F$ and $G$ are $\vdash$-filters of an algebra $\boldsymbol{A}$, and $\theta$ is a congruence of $A$ : if $F \subseteq G$ and $\theta$ is compatible with $F$, then $\theta$ is compatible with $G$.

Numerous additional characterizations of protoalgebraicity are known, e.g., see $[15,20]$. The process of filter generation in algebras is very complicated in general, but it improves as follows in the protoalgebraic case: 
Lemma 2.5. ([11, Thm.3.1]) Let $\vdash$ be protoalgebraic, and let $\boldsymbol{A}$ be an algebra, with $Y \cup\{a\} \subseteq A$.

Then $a \in \mathrm{Fg}_{\vdash}^{\boldsymbol{A}} Y$ iff there exist $\Gamma \cup\{\alpha\} \subseteq F m$ and a homomorphism $h: \boldsymbol{F m} \rightarrow \boldsymbol{A}$ such that $\Gamma \vdash \alpha$ and $h[\Gamma] \subseteq Y \cup \mathrm{Fg}_{\vdash}^{\boldsymbol{A}} \emptyset$ and $h(\alpha)=a$.

The implication from right to left is obvious (regardless of protoalgebraicity), but the forward implication fails in some non-protoalgebraic systems (see [11, Ex. 3.1]).

\section{The Inconsistency Lemma}

A set $\Xi$ of formulas of $\vdash$ is said to be inconsistent in $\vdash$ if $\Xi \vdash \alpha$ for all $\alpha \in F m$. Observe that

(5) if $\Xi$ is finite and inconsistent in $\vdash$, then $\Xi \dashv \vdash h[\Xi]$ for all substitutions $h$, because infinitely many variables are at our disposal. The equivalence of the following conditions is almost immediate. (To infer (iv) from (iii), use (5).)

(i) $\vdash$ has a greatest compact theory

(ii) $F m$ is a compact $\vdash$-theory

(iii) some finite set of formulas is inconsistent in $\vdash$

(iv) some finite subset of $F m(1)$ is inconsistent in $\vdash$

(v) there is a finite $\Xi \subseteq F m(1)$ such that $A=\mathrm{Fg}_{\vdash}^{\boldsymbol{A}} \Xi^{\boldsymbol{A}}(a)$ for every algebra $\boldsymbol{A}$ and all $a \in A$.

Recall that in classical and intuitionistic propositional logic, the theory Fm is compact. The finite generating sets for $F m$ include $\Xi=\left\{v_{1}, \neg v_{1}\right\}$ and, in the latter case, $\Xi=\{\perp\}$.

Definition 3.1. Let $\Psi_{n} \subseteq F m(n)$ for all $n \in \mathbb{N}$. We call $\left\{\Psi_{n}: n \in \mathbb{N}\right\}$ an $I L$-sequence for $\vdash$ provided that, whenever $\Gamma \cup\left\{\alpha_{1}, \ldots, \alpha_{n}\right\} \subseteq F m$, then

$\Gamma \cup\left\{\alpha_{1}, \ldots, \alpha_{n}\right\}$ is inconsistent in $\vdash$ iff $\Gamma \vdash \Psi_{n}\left(\alpha_{1}, \ldots, \alpha_{n}\right)$.

Remark 3.2. Suppose $\left\{\Psi_{n}: n \in \mathbb{N}\right\}$ an IL-sequence for $\vdash$. Then

$$
\Psi_{n}\left(\alpha_{1}, \ldots, \alpha_{n}\right) \cup\left\{\alpha_{1}, \ldots, \alpha_{n}\right\} \text { is inconsistent in } \vdash
$$

for all $n \in \mathbb{N}$ and $\alpha_{1}, \ldots, \alpha_{n} \in F m$, as $\Psi_{n}\left(\alpha_{1}, \ldots, \alpha_{n}\right) \vdash \Psi_{n}\left(\alpha_{1}, \ldots, \alpha_{n}\right)$. Since the elements of $\left\{\alpha_{1}, \ldots, \alpha_{n}\right\}$ are not ordered, it follows from (6) and Definition 3.1 that

$$
\Psi_{n}\left(\alpha_{1}, \ldots, \alpha_{n}\right) \dashv \vdash \Psi_{n}\left(\alpha_{f 1}, \ldots, \alpha_{f n}\right) \text { for any permutation } f \text { of } 1, \ldots, n \text {. }
$$

On similar grounds, if $\left\{\Phi_{n}: n \in \mathbb{N}\right\}$ is another IL-sequence for $\vdash$, then $\Psi_{n} \dashv \vdash \Phi_{n}$ for all $n$.

Remark 3.3. It is easy to see that $\emptyset$ occurs in some IL-sequence for $\vdash$ iff $v_{1} \vdash v_{2}$, iff $\{\emptyset: n \in \mathbb{N}\}$ is an IL-sequence for $\vdash$. In this case, every algebra $\boldsymbol{A}$ has at most two $\vdash$-filters ( $A$ and possibly $\emptyset$ ), whence every $\vdash$-filter of $\boldsymbol{A}$ is compact. 
Definition 3.4. An IL-sequence $\left\{\Psi_{n}: n \in \mathbb{N}\right\}$ for $\vdash$ is said to be elementary if it consists of finite sets $\Psi_{n}$. We say that $\vdash$ has an inconsistency lemmabriefly an $I L$ - if it has an elementary IL-sequence.

This definition encompasses the familiar inconsistency lemma (1) of intuitionistic (and classical) propositional logic, because (1) amounts to the conjunction, over all $n \in \mathbb{N}$, of the claims

$$
\Gamma \cup\left\{\alpha_{1}, \ldots, \alpha_{n}\right\} \text { is inconsistent iff } \Gamma \vdash \neg\left(\alpha_{1} \wedge \ldots \wedge \alpha_{n}\right) \text {. }
$$

Our convention that $0 \notin \mathbb{N}$ is significant. (Without it, the constant-free formulation of classical logic would have no inconsistency lemma.) Definitions 3.1 and 3.4 could be streamlined in the case of systems with a binary connective $\wedge$ such that $v_{1}, v_{2} \dashv \vdash v_{1} \wedge v_{2}$. But some systems with an IL have no such connective, e.g.,

$$
\left\{\left\{v_{1} \rightarrow v_{2} \rightarrow \ldots \rightarrow v_{n} \rightarrow \perp\right\}: n \in \mathbb{N}\right\}
$$

is an IL-sequence for the $\rightarrow, \perp$ fragment of intuitionistic logic. ${ }^{1}$

Remark 3.5. When $\vdash$ has an IL-sequence $\left\{\Psi_{n}: n \in \mathbb{N}\right\}$, then it has an inconsistency lemma iff it has a greatest compact theory. Indeed, (6) shows that $\Psi_{1} \cup\left\{v_{1}\right\} \dashv F m$, i.e., $F m=F_{\circ}\left(\Psi_{1} \cup\left\{v_{1}\right\}\right)$. Thus, $F m$ is compact if $\Psi_{1}$ can be chosen finite. Conversely, if some finite $\Xi \subseteq F m(1)$ is inconsistent in $\vdash$, then for each $n \in \mathbb{N}$, there is a finite $\Psi_{n}^{\prime} \subseteq \Psi_{n}$ such that $\Psi_{n}^{\prime} \cup\left\{v_{1}, \ldots, v_{n}\right\} \vdash \Xi$, because $\vdash$ is finitary. This shows that each $\Psi_{n}^{\prime} \cup\left\{v_{1}, \ldots, v_{n}\right\}$ is inconsistent in $\vdash$, whence $\left\{\Psi_{n}^{\prime}: n \in \mathbb{N}\right\}$ is an elementary IL-sequence for $\vdash$, in view of (5). ${ }^{2}$

If $\left\{\Psi_{n}: n \in \mathbb{N}\right\}$ is an elementary IL-sequence for $\vdash$, then for any $\vdash-$ filter $F$ of the algebra $\boldsymbol{F m}$ and any $\alpha_{1}, \ldots, \alpha_{n} \in F m$, we clearly have

$$
F m=F+\mathrm{Fg}_{\vdash}\left\{\alpha_{1}, \ldots, \alpha_{n}\right\} \text { iff } \Psi_{n}\left(\alpha_{1}, \ldots, \alpha_{n}\right) \subseteq F .
$$

The next theorem extends this equivalence from $\boldsymbol{F} \boldsymbol{m}$ to all algebras, but its proof appears to require the assumption of protoalgebraicity.

Theorem 3.6. Let $\left\{\Psi_{n}: n \in \mathbb{N}\right\}$ be an elementary IL-sequence for a protoalgebraic deductive system $\vdash$. Let $F$ be $a \vdash$-filter of an algebra $\boldsymbol{A}$, and let $a_{1}, \ldots, a_{n} \in A$, where $n \in \mathbb{N}$. Then

$$
A=F+{ }^{\boldsymbol{A}} \mathrm{Fg}_{\vdash}^{\boldsymbol{A}}\left\{a_{1}, \ldots, a_{n}\right\} \text { iff } \Psi_{n}^{\boldsymbol{A}}\left(a_{1}, \ldots, a_{n}\right) \subseteq F .
$$

\footnotetext{
${ }^{1}$ Here and subsequently, suppressed parentheses in iterated implications associate to the right, e.g., $\gamma \rightarrow \alpha \rightarrow \beta$ means $\gamma \rightarrow(\alpha \rightarrow \beta)$. In accommodating examples of this kind, our notion of an IL contrasts with the condition called 'PIRA' in [19].

${ }^{2}$ Of course, when $\vdash$ does not have a greatest compact theory, it can be extended to one that does, by adding a new constant $\perp$ and an inference rule $\perp / v_{1}$ to any given axiomatization. This move creates no new theorems or derivable rules in the old vocabulary.
} 
Proof. By Remark 3.5, some finite $\Xi \subseteq F m(1)$ is inconsistent in $\vdash$, and by Remark 3.2,

$$
\Psi_{n}, v_{1}, \ldots, v_{n} \vdash \Xi\left(v_{n+1}\right) .
$$

By (7), and because $A=\operatorname{Fg}_{\vdash}^{A} \Xi^{\boldsymbol{A}}(a)$ for all $a \in A$, it suffices to prove the forward implication in the present theorem's statement.

Let $a \in A$ and suppose $A=F+\operatorname{Fg}_{\vdash}^{A}\left\{a_{1}, \ldots, a_{n}\right\}$, i.e., $\Xi^{\boldsymbol{A}}(a) \subseteq F+$ $\operatorname{Fg}_{\vdash}^{A}\left\{a_{1}, \ldots, a_{n}\right\}$. For each $\xi \in \Xi$, Lemma 2.5 shows that there exist $\Gamma_{\xi} \cup$ $\left\{\beta_{\xi}\right\} \subseteq F m$ and a homomorphism $h_{\xi}: \boldsymbol{F m} \rightarrow \boldsymbol{A}$ such that

$$
\Gamma_{\xi} \vdash \beta_{\xi} \text { and } h_{\xi}\left[\Gamma_{\xi}\right] \subseteq F \cup\left\{a_{1}, \ldots, a_{n}\right\} \text { and } h_{\xi}\left(\beta_{\xi}\right)=\xi^{\boldsymbol{A}}(a) \text {. }
$$

As $\vdash$ is finitary, we may assume that each $\Gamma_{\xi}$ is finite. Then, by substitutioninvariance, we can arrange that $\Gamma_{\xi} \cup\left\{\beta_{\xi}\right\}$ and $\Gamma_{\xi^{\prime}} \cup\left\{\beta_{\xi^{\prime}}\right\}$ involve disjoint sets of variables whenever $\xi \neq \xi^{\prime} \in \Xi$, and that all of these variables are among $x_{1}, x_{2}, x_{3}, \ldots$. Because of this, we can choose a single homomorphism $h: \boldsymbol{F m} \rightarrow \boldsymbol{A}$ that agrees with $h_{\xi}$ on the variables of $\Gamma_{\xi} \cup\left\{\beta_{\xi}\right\}$ for each $\xi \in \Xi$, and we can also arrange that $h\left(v_{i}\right)=a_{i}$ for $i=1, \ldots, n$ and $h\left(v_{n+1}\right)=a$. We now have

$$
\bigcup_{\xi \in \Xi} \Gamma_{\xi} \vdash\left\{\beta_{\xi}: \xi \in \Xi\right\} \text { and } h\left[\bigcup_{\xi \in \Xi} \Gamma_{\xi}\right] \subseteq F \cup\left\{a_{1}, \ldots, a_{n}\right\} .
$$

Choose a finite $\Lambda \subseteq F m(2)$ as in Definition 2.3. For each $\xi \in \Xi$, it follows from (4) that

$$
\Lambda\left(\beta_{\xi}, \xi\left(v_{n+1}\right)\right), \beta_{\xi} \vdash \xi\left(v_{n+1}\right),
$$

and for all $\lambda \in \Lambda$,

$$
h\left(\lambda\left(\beta_{\xi}, \xi\left(v_{n+1}\right)\right)\right)=\lambda^{\boldsymbol{A}}\left(h\left(\beta_{\xi}\right), h\left(\xi\left(v_{n+1}\right)\right)\right)=\lambda^{\boldsymbol{A}}\left(\xi^{\boldsymbol{A}}(a), \xi^{\boldsymbol{A}}(a)\right) \in F,
$$

by (3). So, if

$$
\Gamma:=\left(\bigcup_{\xi \in \Xi} \Gamma_{\xi}\right) \cup\left(\bigcup_{\xi \in \Xi} \Lambda\left(\beta_{\xi}, \xi\left(v_{n+1}\right)\right)\right) \cup\left\{v_{1}, \ldots, v_{n}\right\},
$$

then $\Gamma \vdash \Xi\left(v_{n+1}\right)$ (by (8)) and $h[\Gamma] \subseteq F \cup\left\{a_{1}, \ldots, a_{n}\right\}$. Now

$$
\Gamma=\Gamma^{\prime} \cup \Pi_{1} \cup \ldots \cup \Pi_{n} \text {, say, }
$$

where $h\left[\Gamma^{\prime}\right] \subseteq F$ and $v_{i} \in \Pi_{i}$ and $h\left[\Pi_{i}\right]=\left\{a_{i}\right\}$ for $i=1, \ldots, n$.

For each $i$ and each $\alpha \in \Pi_{i}$, we also have

$$
\Lambda\left(v_{i}, \alpha\right), v_{i} \vdash \alpha,
$$

by (4), and $h\left(v_{i}\right)=h(\alpha)$, so we may assume without loss of generality that $\Pi_{i}=\left\{v_{i}\right\}$. Indeed, $h\left[\Gamma^{\prime}\right] \subseteq F$ remains true if we add every

$$
\lambda\left(v_{i}, \alpha\right)\left(\lambda \in \Lambda, v_{i} \neq \alpha \in \Pi_{i}, 1 \leq i \leq n\right)
$$

to $\Gamma^{\prime}$ (by (3)), whereupon $\Gamma \vdash \Xi\left(v_{n+1}\right)$ survives if we delete every element other than $v_{i}$ from each $\Pi_{i}$ (by (9)).

In other words, we can arrange that $\Gamma=\left\{\gamma_{1}, \ldots, \gamma_{r}, v_{1}, \ldots, v_{n}\right\}$, where $h$ sends $\gamma_{1}, \ldots, \gamma_{r}$ into $F$. Now $\Gamma$ is inconsistent in $\vdash$ (because $\Gamma \vdash \Xi\left(v_{n+1}\right)$ ), 
so $\gamma_{1}, \ldots, \gamma_{r} \vdash \Psi_{n}$, by the IL. Then, since $h\left[\left\{\gamma_{1}, \ldots, \gamma_{r}\right\}\right] \subseteq F$, we have $\Psi_{n}^{\boldsymbol{A}}\left(a_{1}, \ldots, a_{n}\right)=h\left[\Psi_{n}\right] \subseteq F$, as required.

A deductive system $\vdash$ is said to have a deduction-detachment theorem (DDT) if, for some fixed family $\Sigma$ of binary formulas, the law

$$
\Gamma \cup\{\alpha\} \vdash \beta \quad \text { iff } \Gamma \vdash \Sigma(\alpha, \beta)
$$

applies to all sets of formulas $\Gamma \cup\{\alpha, \beta\}$. In this case, $\vdash$ is protoalgebraic and $\Sigma$ can be chosen finite, as $\vdash$ is finitary. The inconsistency lemma of classical or intuitionistic propositional logic is a corollary of the standard DDT for these systems, viz. $\Gamma \cup\{\alpha\} \vdash \beta$ iff $\Gamma \vdash \alpha \rightarrow \beta$. In the introduction, we mentioned a semantic characterization of the DDT, given in [12]. We can now characterize the inconsistency lemma in an analogous way and, thanks to Theorem 3.6, a somewhat similar argument can be used. First, we must recall some notions from lattice theory:

An idempotent commutative semigroup $\langle S ;+\rangle$ is called a join semilattice with 0 if it has a least element with respect to the order

$$
x \leq y \Longleftrightarrow x+y=y .
$$

It is then said to be dually pseudo-complemented if it has a greatest element 1 and, for each $a \in S$, there is a smallest $b \in S$ such that $a+b=1$. This $b$ is denoted as $a^{*}$. Observe that $a^{* *} \leq a$ for all $a \in S$.

Clearly, the compact $\vdash$-filters of an algebra $\boldsymbol{A}$ form a join semilattice with 0 under the operation $+{ }^{\boldsymbol{A}}$, and the semilattice order $\leq$ is just $\subseteq$.

Theorem 3.7. Let $\vdash$ be a protoalgebraic deductive system. Then the following conditions are equivalent.

(i) $\vdash$ has an inconsistency lemma.

(ii) For every algebra $\boldsymbol{A}$, the compact $\vdash$-filters of $\boldsymbol{A}$ form a dually pseudo-complemented semilattice with respect to $+{ }^{\boldsymbol{A}}$.

(iii) The join semilattice of compact $\vdash$-theories is dually pseudo-complemented.

Proof. We may assume that $F m$ is a compact $\vdash-$ theory, as this is entailed by all three conditions (see Remark 3.5). In other words, some finite $\Xi \subseteq F m(1)$ is inconsistent in $\vdash$.

(i) $\Rightarrow$ (ii): Let $\left\{\Psi_{n}: n \in \mathbb{N}\right\}$ be an elementary IL-sequence for $\vdash$. Let $a_{1}, \ldots, a_{n}$ be elements of an algebra $\boldsymbol{A}$, where $n \in \mathbb{N}$, and let $H=$ $\operatorname{Fg}_{\vdash} \boldsymbol{A}\left\{a_{1}, \ldots, a_{n}\right\}$. For any $\vdash-$ filter $F$ of $\boldsymbol{A}$, we have

$$
A=F+{ }^{\boldsymbol{A}} H \text { iff } \Psi_{n}^{\boldsymbol{A}}\left(a_{1}, \ldots, a_{n}\right) \subseteq F,
$$

by Theorem 3.6. So, because $\Psi_{n}$ is finite, $\operatorname{Fg}_{\vdash}^{\boldsymbol{A}} \Psi_{n}^{\boldsymbol{A}}\left(a_{1}, \ldots, a_{n}\right)$ is compact and equal to $H^{*}$ in the semilattice of compact $\vdash$-filters of $\boldsymbol{A}$. Finally, if $\emptyset$ is a $\vdash$-filter of $\boldsymbol{A}$, then $\emptyset^{*}=A$, which is also compact, because $\vdash$ has a greatest compact theory. 
(ii) $\Rightarrow$ (iii) is trivial.

(iii) $\Rightarrow$ (i): Let $n \in \mathbb{N}$ and choose a finite $\Psi_{n}^{\prime} \subseteq F m$ so that

$$
\mathrm{Fg}_{\vdash} \Psi_{n}^{\prime}=\left(\mathrm{Fg}_{\vdash}\left\{v_{1}, \ldots, v_{n}\right\}\right)^{*}
$$

in the semilattice of compact $\vdash-$ theories. Let

$$
\Psi_{n}=g\left[\Psi_{n}^{\prime}\right],
$$

where $g$ is a substitution that fixes $v_{1}, \ldots, v_{n}$ and sends all other variables to $v_{1}$, so $\Psi_{n}$ is a finite subset of $F m(n)$. For any $\vdash$-theory $F$ (compact or not), we have

$$
F m=F+\mathrm{Fg}_{\vdash}\left\{v_{1}, \ldots, v_{n}\right\} \quad \text { iff } \mathrm{Fg}_{\vdash} \Psi_{n}^{\prime} \subseteq F \quad \text { (i.e., } \Psi_{n}^{\prime} \subseteq F \text { ). }
$$

This is due to the compactness of $F m$ and the fact that $F$ is a join of compact elements of the lattice of all $\vdash$-theories, because this lattice is algebraic.

Given $\Gamma \cup\left\{\alpha_{1}, \ldots, \alpha_{n}\right\} \subseteq F m$, let $h$ be a surjective substitution that sends $v_{i}$ to $\alpha_{i}$ for $i=1, \ldots, n$ and that sends to $v_{1}$ all other variables occurring in $\Psi_{n}^{\prime}$. Then $h\left[\Psi_{n}^{\prime}\right]=\Psi_{n}\left(\alpha_{1}, \ldots, \alpha_{n}\right)$ and $\Gamma=h\left[h^{-1}[\Gamma]\right]$. It suffices to show that $\Gamma \cup\left\{\alpha_{1}, \ldots, \alpha_{n}\right\}$ is inconsistent in $\vdash$ iff $\Gamma \vdash \Psi_{n}\left(\alpha_{1}, \ldots, \alpha_{n}\right)$.

By Lemma 2.2(i), $h^{-1}\left[\mathrm{Fg}_{\vdash} \Gamma\right]$ and $h^{-1}\left[\mathrm{Fg}_{\vdash}\left(\Gamma \cup\left\{\alpha_{1}, \ldots, \alpha_{n}\right\}\right)\right]$ are $\vdash-$ theories, and ker $h$ is obviously compatible with $h^{-1}\left[\mathrm{Fg}_{\vdash} \Gamma\right]$. By protoalgebraicity and Theorem 2.4, ker $h$ is also compatible with the larger theory

$$
Y:=h^{-1}\left[\mathrm{Fg}_{\vdash} \Gamma\right]+\mathrm{Fg}_{\vdash}\left\{v_{1}, \ldots, v_{n}\right\}=\mathrm{Fg}_{\vdash}\left(h^{-1}\left[\mathrm{Fg}_{\vdash} \Gamma\right] \cup\left\{v_{1}, \ldots, v_{n}\right\}\right) .
$$

Therefore, $h[Y]$ is a $\vdash$-theory, by Lemma 2.2(ii). It follows that $h[Y]$ contains $\mathrm{Fg}_{\vdash}\left(\Gamma \cup\left\{\alpha_{1}, \ldots, \alpha_{n}\right\}\right)$, because it contains

$$
h\left[h^{-1}[\Gamma] \cup\left\{v_{1}, \ldots, v_{n}\right\}\right]=\Gamma \cup\left\{\alpha_{1}, \ldots, \alpha_{n}\right\} .
$$

On the other hand, it is clear that $Y \subseteq h^{-1}\left[\mathrm{Fg}_{\vdash}\left(\Gamma \cup\left\{\alpha_{1}, \ldots, \alpha_{n}\right\}\right)\right]$, so

$$
h[Y]=\mathrm{Fg}_{\vdash}\left(\Gamma \cup\left\{\alpha_{1}, \ldots, \alpha_{n}\right\}\right) .
$$

Now $\Gamma \cup\left\{\alpha_{1}, \ldots, \alpha_{n}\right\}$ is inconsistent in $\vdash$ iff $\Gamma, \alpha_{1}, \ldots, \alpha_{n} \vdash \Xi$,

iff $\Gamma, \alpha_{1}, \ldots, \alpha_{n} \vdash h[\Xi] \quad($ as $\Xi \dashv \vdash h[\Xi])$,

iff $h[\Xi] \subseteq h[Y]$ (by (11)),

iff $\Xi \subseteq Y$ (because ker $h$ is compatible with $Y$ ),

iff $F m=h^{-1}\left[\mathrm{Fg}_{\vdash} \Gamma\right]+\mathrm{Fg}_{\vdash}\left\{v_{1}, \ldots, v_{n}\right\}$,

iff $\Psi_{n}^{\prime} \subseteq h^{-1}\left[\mathrm{Fg}_{\vdash} \Gamma\right]$ (by (10)),

iff $h\left[\Psi_{n}^{\prime}\right] \subseteq \mathrm{Fg}_{\vdash} \Gamma$,

iff $\Gamma \vdash h\left[\Psi_{n}^{\prime}\right]$, i.e., $\Gamma \vdash \Psi_{n}\left(\alpha_{1}, \ldots, \alpha_{n}\right)$.

Corollary 3.8. If a protoalgebraic deductive system $\vdash$ has an inconsistency lemma, then every finite algebra has a dually pseudo-complemented lattice of $\vdash$-filters. 
A join semilattice $\langle S ;+\rangle$ with 0 is said to be dually Brouwerian if, for any $a, b \in S$, there is a smallest $c \in S$ such that $a \leq b+c$. As we recounted in the introduction, a deductive system has a DDT iff it is protoalgebraic and has a dually Brouwerian join semilattice of compact theories [12] (see [10] also). A dually Brouwerian join semilattice with 0 is dually pseudo-complemented if it has a greatest element, so the next result follows from Theorem 3.7.

Corollary 3.9. If a deductive system with a greatest compact theory has a deduction-detachment theorem, then it has an inconsistency lemma.

A syntactic proof is also possible. The converse of Corollary 3.9 is false, even for protoalgebraic systems (see the discussion of $\boldsymbol{\Pi}$ in Section 6).

A deductive system with an inconsistency lemma need not be protoalgebraic, e.g., the usual IL of intuitionistic propositional logic (IPL) persists in the implication-less fragment of IPL (i.e., the $\wedge, \vee, \neg, \perp, \top$ fragment), which is no longer protoalgebraic [8]. The proof of Theorem 3.7 makes considerable use of protoalgebraicity, however, and it is not clear how to prove it in the absence of that assumption. On the other hand, every algebraizable deductive system is protoalgebraic. We discuss this case below.

Given a quasivariety $\mathrm{K}$ and an algebra $\boldsymbol{A}$ of the same type, the $\mathrm{K}$ congruences of $\boldsymbol{A}$ are the congruences $\theta$ such that $\boldsymbol{A} / \theta \in \mathrm{K}$. They form an algebraic closure system over $A \times A$, and hence an algebraic lattice, ordered by inclusion, in which the compact elements are just the finitely generated $\mathrm{K}$-congruences. At the same time, for any subset $F$ of $\boldsymbol{A}$, there is always a largest congruence of $\boldsymbol{A}$ that is compatible with $F$; it is denoted by $\Omega^{\boldsymbol{A}} F$. A deductive system $\vdash$ is said to be algebraized by $\mathrm{K}$ if, for every algebra $\boldsymbol{A}$, the rule $F \mapsto \Omega^{\boldsymbol{A}} F$ defines an isomorphism from the $\vdash$-filter lattice of $\boldsymbol{A}$ onto the lattice of $\mathrm{K}$-congruences of $\boldsymbol{A}$. We say that $\vdash$ is (elementarily) algebraizable if it is algebraized by some quasivariety $\mathrm{K}$. In this case $\mathrm{K}$ is unique, and if $\mathrm{K}$ is a variety, then $\vdash$ is said to be strongly algebraizable. These definitions are equivalent to the original syntactic ones, see Blok and Pigozzi [8].

For every quasivariety $\mathrm{K}$, the $\mathrm{K}$-congruence lattices of $\boldsymbol{A}$ and $\boldsymbol{A} / \mathrm{Cg}_{\mathrm{K}}^{\boldsymbol{A}} \emptyset$ are isomorphic, where $\operatorname{Cg}_{\mathrm{K}}^{\boldsymbol{A}} Y$ denotes the least $\mathrm{K}$-congruence of $\boldsymbol{A}$ containing a subset $Y$ of $A \times A$. Since $\boldsymbol{A} / \mathrm{Cg}_{\mathrm{K}}^{\boldsymbol{A}} \emptyset \in \mathrm{K}$, the following conclusion can be drawn from Theorem 3.7.

Theorem 3.10. Let $\mathrm{K}$ be a quasivariety that algebraizes a deductive system $\vdash$. Then the following conditions are equivalent.

(i) $\vdash$ has an inconsistency lemma.

(ii) For every algebra $\boldsymbol{A}$, the join semilattice of compact $\mathrm{K}$-congruences of $\boldsymbol{A}$ is dually pseudo-complemented.

(iii) For every $\boldsymbol{A} \in \mathrm{K}$, the join semilattice of compact $\mathrm{K}$-congruences of $\boldsymbol{A}$ is dually pseudo-complemented.

If $\mathrm{K}$ is a variety, then these conditions are equivalent to 
(iv) For every $\boldsymbol{A} \in \mathrm{K}$, the join semilattice of compact congruences of $\boldsymbol{A}$ is dually pseudo-complemented.

\section{Classical Inconsistency Lemmas}

Suppose $\langle S ;+\rangle$ is a dually pseudo-complemented join semilattice with 0 . A well known result of Glivenko [27] shows that the sub-poset $\left\{a^{*}: a \in S\right\}$ of $\langle S ; \leq\rangle$ is a Boolean lattice, i.e., a complemented distributive lattice (see $[28$, p. 49] also). Of course, the join operation of this lattice is + . The meet operation $\cdot$ is given by $a \cdot b=\left(a^{*}+b^{*}\right)^{*}$. In particular, if $\langle S ;+\rangle$ satisfies $x^{* *}=x$, then it is a Boolean lattice with respect to the join semilattice order. The converse is true as well, because in a Boolean lattice, (dual) pseudo-complements coincide with complements and the latter are unique.

Note, however, that when the compact $\vdash$-filters of an infinite algebra form a Boolean lattice, they don't always form a sublattice of the lattice of all $\vdash$-filters, because the intersection of two compact $\vdash$-filters need not be compact.

In classical propositional logic, the familiar inconsistency lemma (2) can be formulated equivalently as the conjunction, over $n \in \mathbb{N}$, of the assertions

$$
\Gamma \cup\left\{\neg\left(\alpha_{1} \wedge \ldots \wedge \alpha_{n}\right)\right\} \text { is inconsistent iff } \Gamma \vdash\left\{\alpha_{1}, \ldots, \alpha_{n}\right\} .
$$

Of course, (2) and (12) are false in the context of intuitionistic logic, where $p \vdash \neg \neg p$ is derivable but $\neg \neg p \vdash p$ is not. For arbitrary deductive systems $\vdash$, we may abstract (12) as follows.

Definition 4.1. An IL-sequence $\left\{\Psi_{n}: n \in \mathbb{N}\right\}$ for $\vdash$ will be called classical provided that, whenever $n \in \mathbb{N}$ and $\Gamma \cup\left\{\alpha_{1}, \ldots, \alpha_{n}\right\} \subseteq F m$, then

$\Gamma \cup \Psi_{n}\left(\alpha_{1}, \ldots, \alpha_{n}\right)$ is inconsistent in $\vdash$ iff $\Gamma \vdash\left\{\alpha_{1}, \ldots, \alpha_{n}\right\}$.

We say that $\vdash$ has a classical inconsistency lemma if it has a classical elementary IL-sequence.

Remark 3.2 shows that when $\vdash$ has a classical IL-sequence, then every ILsequence for $\vdash$ is classical. It is easily verified that a [classical] IL-sequence for $\vdash$ remains a [classical] IL-sequence for any axiomatic extension of $\vdash$ (in the same language).

Suppose $\left\{\Psi_{n}: n \in \mathbb{N}\right\}$ is an elementary IL-sequence for $\vdash$, where no $\Psi_{n}$ is empty. For each $n \in \mathbb{N}$, let $\# n=\left|\Psi_{n}\right|$ and let $\Psi_{n}=\left\{\psi_{n}^{1}, \ldots, \psi_{n}^{\# n}\right\}$. Put

$$
\Psi_{\# n} \Psi_{n}:=\Psi_{\# n}\left(\psi_{n}^{1}, \ldots, \psi_{n}^{\# n}\right) .
$$

Each $\Psi_{\# n} \Psi_{n}$ is a well defined (finite non-empty) subset of $F m(n)$, by the observation about permutations in Remark 3.2. For any $\alpha_{1}, \ldots, \alpha_{n} \in F m$, we have

$$
\alpha_{1}, \ldots, \alpha_{n} \vdash \Psi_{\# n} \Psi_{n}\left(\alpha_{1}, \ldots, \alpha_{n}\right),
$$


because $\left\{\alpha_{1}, \ldots, \alpha_{n}\right\} \cup \Psi_{n}\left(\alpha_{1}, \ldots, \alpha_{n}\right)$ is inconsistent in $\vdash$. Writing $\bar{\alpha}$ for $\alpha_{1}, \ldots, \alpha_{n}$, we deduce from $\Psi_{\# n} \Psi_{n}(\bar{\alpha}) \vdash \Psi_{\# n} \Psi_{n}(\bar{\alpha})$ that

$$
\Psi_{\# n} \Psi_{n}(\bar{\alpha}) \cup \Psi_{n}(\bar{\alpha}) \text { is inconsistent in } \vdash \text {. }
$$

In the classical case, this entails an abstraction of $p \dashv \vdash \neg \neg p$ :

Lemma 4.2. Let $\left\{\Psi_{n}: n \in \mathbb{N}\right\}$ be an elementary IL-sequence for $\vdash$, with $\Psi_{n} \neq \emptyset$ for all $n$. This sequence is classical iff, for any formulas $\alpha_{1}, \ldots, \alpha_{n}$ $(n \in \mathbb{N})$, we have

$$
\alpha_{1}, \ldots, \alpha_{n} \dashv \Vdash \Psi_{\# n} \Psi_{n}\left(\alpha_{1}, \ldots, \alpha_{n}\right) .
$$

In that case, whenever $a_{1}, \ldots, a_{n}$ are elements of an algebra $\boldsymbol{A}$, then

$$
\mathrm{Fg}_{\vdash}^{\boldsymbol{A}}\left\{a_{1}, \ldots, a_{n}\right\}=\mathrm{Fg}_{\vdash}^{\boldsymbol{A}} \Psi_{\# n}^{\boldsymbol{A}} \Psi_{n}^{\boldsymbol{A}}\left(a_{1}, \ldots, a_{n}\right) .
$$

Proof. In the forward direction, (14) yields $\Psi_{\# n} \Psi_{n}(\bar{\alpha}) \vdash\left\{\alpha_{1}, \ldots, \alpha_{n}\right\}$, because $\left\{\Psi_{n}: n \in \mathbb{N}\right\}$ is classical. This, with (13), gives (15), hence the second claim of the lemma. Conversely, (15) allows us to reformulate the criterion in Definition 4.1 as

$\Gamma \cup \Psi_{n}\left(\alpha_{1}, \ldots, \alpha_{n}\right)$ is inconsistent in $\vdash$ iff $\Gamma \vdash \Psi_{\# n} \Psi_{n}\left(\alpha_{1}, \ldots, \alpha_{n}\right)$, the truth of which follows from the (ordinary) IL.

Theorem 4.3. Let $\vdash$ be a protoalgebraic deductive system. Then the following conditions are equivalent.

(i) $\vdash$ has a classical inconsistency lemma.

(ii) For every algebra $\boldsymbol{A}$, the compact $\vdash$-filters of $\boldsymbol{A}$ form a Boolean sublattice of the lattice of all $\vdash$-filters of $\boldsymbol{A}$.

(iii) The join semilattice of compact $\vdash$-theories is a Boolean lattice.

In this case, every finite algebra has a Boolean lattice of $\vdash$-filters.

Proof. If $v_{1} \vdash v_{2}$, then $\{\emptyset: n \in \mathbb{N}\}$ is a classical elementary IL-sequence for $\vdash$ and all three conditions hold (see Remark 3.3). We may therefore assume that $v_{1} \nvdash v_{2}$; equivalently, $\emptyset$ does not occur in any IL-sequence for $\vdash$. Now the set $\Lambda$ in Definition 2.3 cannot be empty, by (4). So, by (3), $\emptyset$ is not a $\vdash$-filter of any algebra.

(i) $\Rightarrow$ (ii): Let $\left\{\Psi_{n}: n \in \mathbb{N}\right\}$ be a classical elementary IL-sequence for $\vdash$, and let $H$ be a compact $\vdash$-filter of an algebra $\boldsymbol{A}$. Since $H \neq \emptyset$, it has the form $\operatorname{Fg}_{\vdash}^{\boldsymbol{A}}\left\{a_{1}, \ldots, a_{n}\right\}$, where $n \in \mathbb{N}$. In the join semilattice of compact $\vdash-$ filters of $\boldsymbol{A}$, we have $H^{*}=\operatorname{Fg}_{\vdash}^{\boldsymbol{A}} \Psi_{n}^{\boldsymbol{A}}\left(a_{1}, \ldots, a_{n}\right)$, by the proof of Theorem 3.7. Because $\Psi_{n} \neq \emptyset$, the same argument shows that

$$
H^{* *}=\mathrm{Fg}_{\vdash}^{\boldsymbol{A}} \Psi_{\# n}^{\boldsymbol{A}} \Psi_{n}^{\boldsymbol{A}}\left(a_{1}, \ldots, a_{n}\right),
$$

so $H^{* *}=H$, by (16). Thus, the compact $\vdash-$ filters of $\boldsymbol{A}$ form a Boolean lattice with respect to $\subseteq$, by the result of Glivenko mentioned at the beginning of this section. It remains to show that the intersection of any two compact 
$\vdash$-filters of $\boldsymbol{A}$ is compact. Let $\bar{a}=a_{1}, \ldots, a_{n} \in A$ and $\bar{b}=b_{1}, \ldots, b_{m} \in A$, where $n, m \in \mathbb{N}$ (so $\Psi_{n}, \Psi_{m} \neq \emptyset$ ). We claim that

$$
\operatorname{Fg}_{\vdash}^{\boldsymbol{A}}\{\bar{a}\} \cap \mathrm{Fg}_{\vdash}^{\boldsymbol{A}}\{\bar{b}\}=\mathrm{Fg}_{\vdash}^{\boldsymbol{A}} \Psi_{\# n+\# m}^{\boldsymbol{A}}\left(\Psi_{n}^{\boldsymbol{A}}(\bar{a}), \Psi_{m}^{\boldsymbol{A}}(\bar{b})\right),
$$

where the right hand side abbreviates

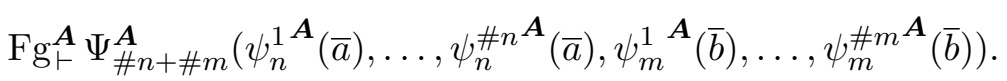

Indeed, $A=\mathrm{Fg}_{\vdash}^{\boldsymbol{A}}\{\bar{a}\}+{ }^{\boldsymbol{A}} \mathrm{Fg}_{\vdash}^{\boldsymbol{A}} \Psi_{n}^{\boldsymbol{A}}(\bar{a})=\mathrm{Fg}_{\vdash}^{\boldsymbol{A}}\{\bar{b}\}+{ }^{\boldsymbol{A}} \mathrm{Fg}_{\vdash}^{\boldsymbol{A}} \Psi_{m}^{\boldsymbol{A}}(\bar{b})$, whence $\boldsymbol{A}=\mathrm{Fg}_{\vdash}^{\boldsymbol{A}}\{\bar{a}\}+{ }^{\boldsymbol{A}} \mathrm{Fg}_{\vdash}^{\boldsymbol{A}}\left(\Psi_{n}^{\boldsymbol{A}}(\bar{a}) \cup \Psi_{m}^{\boldsymbol{A}}(\bar{b})\right)=\mathrm{Fg}_{\vdash}^{\boldsymbol{A}}\{\bar{b}\}+{ }^{\boldsymbol{A}} \mathrm{Fg}_{\vdash}^{\boldsymbol{A}}\left(\Psi_{n}^{\boldsymbol{A}}(\bar{a}) \cup \Psi_{m}^{\boldsymbol{A}}(\bar{b})\right)$, and so $\operatorname{Fg}_{\vdash}^{\boldsymbol{A}} \Psi_{\# n+\# m}^{\boldsymbol{A}}\left(\Psi_{n}^{\boldsymbol{A}}(\bar{a}), \Psi_{m}^{\boldsymbol{A}}(\bar{b})\right) \subseteq \mathrm{Fg}_{\vdash}^{\boldsymbol{A}}\{\bar{a}\} \cap \mathrm{Fg}_{\vdash}^{\boldsymbol{A}}\{\bar{b}\}$, by Theorem 3.6. Conversely, suppose $c \in \operatorname{Fg}_{\vdash}^{A}\{\bar{a}\} \cap \operatorname{Fg}_{\vdash}^{\boldsymbol{A}}\{\bar{b}\}$. Then

$$
\mathrm{Fg}_{\vdash}^{\boldsymbol{A}}\{\bar{a}\}+{ }^{\boldsymbol{A}} \mathrm{Fg}_{\vdash}^{\boldsymbol{A}} \Psi_{1}^{\boldsymbol{A}}(c) \supseteq \mathrm{Fg}_{\vdash}^{\boldsymbol{A}}\{c\}+{ }^{\boldsymbol{A}} \mathrm{Fg}_{\vdash}^{\boldsymbol{A}} \Psi_{1}^{\boldsymbol{A}}(c)=A,
$$

and similarly for $\bar{b}$, so

$$
\boldsymbol{A}=\mathrm{Fg}_{\vdash}^{\boldsymbol{A}}\{\bar{a}\}+{ }^{\boldsymbol{A}} \mathrm{Fg}_{\vdash}^{\boldsymbol{A}} \Psi_{1}^{\boldsymbol{A}}(c)=\mathrm{Fg}_{\vdash}^{\boldsymbol{A}}\{\bar{b}\}+{ }^{\boldsymbol{A}} \mathrm{Fg}_{\vdash}^{\boldsymbol{A}} \Psi_{1}^{\boldsymbol{A}}(c) .
$$

Then $\Psi_{n}^{\boldsymbol{A}}(\bar{a}) \cup \Psi_{m}^{\boldsymbol{A}}(\bar{b}) \subseteq \operatorname{Fg}_{\vdash}^{\boldsymbol{A}} \Psi_{1}^{\boldsymbol{A}}(c)$, by Theorem 3.6 again. Now Boolean lattices satisfy $x \leq y^{*} \Longrightarrow y \leq x^{*}$, so

$$
\mathrm{Fg}_{\vdash}^{\boldsymbol{A}}\{c\} \subseteq \mathrm{Fg}_{\vdash}^{\boldsymbol{A}} \Psi_{\# n+\# m}^{\boldsymbol{A}}\left(\Psi_{n}^{\boldsymbol{A}}(\bar{a}), \Psi_{m}^{\boldsymbol{A}}(\bar{b})\right),
$$

completing the proof of (17). Since the right hand side of (17) is compact, we have proved (ii).

(ii) $\Rightarrow$ (iii) is trivial.

(iii) $\Rightarrow$ (i): By (iii), the semilattice of compact $\vdash-$ theories is dually pseudocomplemented, so by Theorem 3.7 and its proof, $\vdash$ has an elementary IL-sequence $\left\{\Psi_{n}: n \in \mathbb{N}\right\}$ and $\left(\mathrm{Fg}_{\vdash}\left\{\alpha_{1}, \ldots, \alpha_{n}\right\}\right)^{*}=\mathrm{Fg}_{\vdash} \Psi_{n}\left(\alpha_{1}, \ldots, \alpha_{n}\right)$ whenever $\alpha_{1}, \ldots, \alpha_{n} \in F m$. The identity $x^{* *}=x$ shows that (15) holds, so the IL-sequence is classical, by Lemma 4.2.

Corollary 4.4. If a protoalgebraic deductive system has a classical inconsistency lemma, then it has a deduction-detachment theorem.

Proof. This follows from Theorem 4.3 and the analogous characterization of the DDT, because the + reduct of a Boolean lattice $\left\langle B ; \cdot,+,{ }^{*}\right\rangle$ is a dually Brouwerian join semilattice with 0 , in which $a \cdot b^{*}$ is always the least $c$ such that $a \leq b+c$.

Alternatively, we can verify that, whenever $\left\{\Psi_{n}: n \in \omega\right\}$ is a classical elementary IL-sequence for $\vdash$ and $\Gamma \cup\{\alpha, \beta\} \subseteq F m$, then

$$
\Gamma \cup\{\alpha\} \vdash \beta \text { iff } \Gamma \vdash \Psi_{1+\# 1}\left(\alpha, \psi_{1}^{1}(\beta), \ldots, \psi_{1}^{\# 1}(\beta)\right) .
$$

Corollary 4.5. If a protoalgebraic deductive system $\vdash$ has a classical inconsistency lemma, then the $\vdash$-filters of any algebra $\boldsymbol{A}$ form a pseudocomplemented $^{3}$ distributive lattice.

\footnotetext{
${ }^{3}$ i.e., for each $\vdash$-filter $F$, there is a largest $\vdash-$ filter $G$ such that $F \cap G=\operatorname{Fg}_{\vdash}^{\boldsymbol{A}} \emptyset$.
} 
Proof. By Corollary 4.4, $\vdash$ has a DDT, and a DDT always entails filterdistributivity [12]. Because the $\vdash$-filter lattice of $\boldsymbol{A}$ is algebraic, it is isomorphic to the ideal lattice of the join semilattice $\boldsymbol{S}$ of compact $\vdash$-filters of $\boldsymbol{A}$, and $\boldsymbol{S}$ is a Boolean lattice, by Theorem 4.3. But, by another result of Glivenko [27], the ideal lattice of a distributive lattice with 0 is always pseudo-complemented (see [28, p. 91]).

\section{Semisimplicity, EDPRC and Filtrality}

A quasivariety $\mathrm{K}$ is said to be relatively congruence distributive if its members have distributive lattices of $\mathrm{K}$-congruences; it has the relative congruence extension property if every $\mathrm{K}$-congruence on a subalgebra of a member of $\mathrm{K}$ is the restriction of some $\mathrm{K}$-congruence on the parent algebra. An algebra $\boldsymbol{A} \in \mathrm{K}$ is said to be $\mathrm{K}$-subdirectly irreducible or finitely $\mathrm{K}$-subdirectly irreducible or $\mathrm{K}$-simple if, in the $\mathrm{K}$-congruence lattice of $\boldsymbol{A}$, the identity relation $\operatorname{id}_{A}=\{\langle a, a\rangle: a \in A\}$ is completely meet-irreducible or meetirreducible or a co-atom, respectively. Each member of a quasivariety $\mathrm{K}$ is isomorphic to a subdirect product of $\mathrm{K}$-subdirectly irreducible algebras in $\mathrm{K}$ [38, Thm. 1.1]. If every $\mathrm{K}$-subdirectly irreducible algebra in $\mathrm{K}$ is $\mathrm{K}$-simple, then $\mathrm{K}$ is said to be relatively semisimple. We say that $\mathrm{K}$ has equationally definable principal relative congruences (EDPRC) if there exists a finite set $\Phi \subseteq F m(4) \times F m(4)$ such that, whenever $\boldsymbol{A} \in \mathrm{K}$ and $a, b, c, d \in A$, then

$$
\langle c, d\rangle \in \operatorname{Cg}_{\mathrm{K}}^{\boldsymbol{A}}\{\langle a, b\rangle\} \text { iff }\left(\varphi^{\boldsymbol{A}}(a, b, c, d)=\eta^{\boldsymbol{A}}(a, b, c, d) \text { for all }\langle\varphi, \eta\rangle \in \Phi\right) .
$$

In the event that $\mathrm{K}$ is a variety, the congruences and $\mathrm{K}$-congruences of algebras in $\mathrm{K}$ are the same, so the prefixes ' $\mathrm{K}-$ ' and 'relatively' can all be dropped.

Varieties with EDPC were introduced in [22] under a different name; they were studied in a series of papers in Algebra Universalis by Blok, Köhler and Pigozzi (in various author-combinations), published between 1980 and 1994. A variety $\mathrm{K}$ is said to be filtral if every congruence $\theta$ on a subdirect product $\boldsymbol{A}$ of subdirectly irreducible algebras in $\mathrm{K}$ is determined by a suitable filter $X_{\theta}$ over the index set $I$ of the product - that is to say,

$$
\theta=\left\{\langle a, b\rangle \in A \times A:\{i \in I: a(i)=b(i)\} \in X_{\theta}\right\}
$$

(where $X_{\theta}$ consists of subsets of $I$ and is closed under finite intersections and supersets within $I$ ). The filtral varieties turn out to be just the semisimple varieties with EDPC [22], and the discriminator varieties coincide with the congruence permutable filtral varieties (see [4, Cor. 3.4] or [23, Thm. 4.13]).

We use $\mathrm{K}_{\mathrm{FSI}}$ to denote the class of all finitely $\mathrm{K}$-subdirectly irreducible algebras in a quasivariety $\mathrm{K}$. If $\mathrm{K}$ is relatively congruence distributive, then the algebras in $\mathrm{K}_{\mathrm{FSI}}$ are finitely subdirectly irreducible in the absolute sense [18]. The next result is due to Czelakowski and Dziobiak [16, Thm. 2.3]. For varieties, it was proved earlier in [6]. 
Theorem 5.1. The following conditions on a relatively congruence distributive quasivariety $\mathrm{K}$ are equivalent.

(i) $\mathrm{K}_{\mathrm{FSI}}$ is closed under subalgebras and ultraproducts (i.e., it is a universal class).

(ii) For all $\boldsymbol{A} \in \mathrm{K}$, the compact $\mathrm{K}$-congruences of $\boldsymbol{A}$ form a sublattice of the $\mathrm{K}$-congruence lattice of $\boldsymbol{A}$ (i.e., the intersection of any two compact $\mathrm{K}$-congruences is compact).

Corollary 5.2. If an algebra $\boldsymbol{A}$ belongs to a relatively semisimple quasivariety $\mathrm{K}$ with EDPRC, then the compact $\mathrm{K}$-congruences of $\boldsymbol{A}$ form a sublattice of the $\mathrm{K}$-congruence lattice of $\boldsymbol{A}$.

Proof. Every quasivariety with EDPRC is relatively congruence distributive and has the relative congruence extension property [10]. By the latter property, the class of $\mathrm{K}$-simple algebras in $\mathrm{K}$ is closed under nontrivial subalgebras. It is also closed under nontrivial ultraproducts, as a direct consequence of EDPRC, see for instance [15, Prop. Q.9.5]. Whenever Q is the smallest quasivariety containing a class $M$ of similar algebras, then every algebra in $\mathrm{QFSI}_{\mathrm{F}}$ can be embedded into an ultraproduct of members of $\mathrm{M}$, by [16, Lem. 1.5]. So, since $\mathrm{K}$ is relatively semisimple, the nontrivial algebras in $\mathrm{K}_{\mathrm{FSI}}$ are $\mathrm{K}$-simple. Thus, $\mathrm{K}_{\mathrm{FSI}}$ is closed under subalgebras and ultraproducts, and the result follows from Theorem 5.1.

A dual generalized Boolean lattice is a distributive lattice $\langle L ; \cdot,+\rangle$ with a least element 0 such that, for any $a, b \in L$, there exists $c \in L$ with $a \cdot c=0$ and $a+c=a+b$. Note that, in this case, $\langle L ; \cdot,+\rangle$ is a Boolean lattice iff it has a greatest element.

Blok and Pigozzi proved that the join semilattice of compact congruences of an algebra $\boldsymbol{A}$ in a filtral variety $\mathrm{K}$ is always a dual generalized Boolean lattice [5, Cor. 4.3]. Careful inspection of their proof shows that the result remains true for the $\mathrm{K}$-congruences of $\boldsymbol{A}$ when $\mathrm{K}$ is a relatively semisimple quasivariety with EDPRC. Indeed, regardless of semisimplicity, the equations defining the principal relative congruences can be used, as in the case of varieties, to construct another equation that is valid in just those members of $\mathrm{K}$ that have dual generalized Boolean lattices of compact relative congruences. Clearly, this equation holds in the relatively simple members of $\mathrm{K}$, and equations persist in subdirect products. From this, we deduce:

Lemma 5.3. Let $\mathrm{K}$ be a relatively semisimple quasivariety with $E D P R C$, such that, for every $\boldsymbol{A} \in \mathrm{K}$, the total congruence $A \times A$ is compact in the $\mathrm{K}$-congruence lattice of $\boldsymbol{A}$. Then the compact $\mathrm{K}$-congruences of any algebra in $\mathrm{K}$ form a Boolean lattice with respect to $\subseteq$.

Theorem 5.4. Let $\mathrm{K}$ be a quasivariety that algebraizes a deductive system $\vdash$. Then the following conditions are equivalent.

(i) $\vdash$ has a classical inconsistency lemma. 
(ii) $\vdash$ has a greatest compact theory and $\mathrm{K}$ is relatively semisimple with $E D P R C$.

(iii) For every $\boldsymbol{A} \in \mathrm{K}$, the compact $\mathrm{K}$-congruences of $\boldsymbol{A}$ form a Boolean sublattice of the lattice of all $\mathrm{K}$-congruences of $\boldsymbol{A}$.

In this case, the finite algebras in $\mathrm{K}$ have Boolean lattices of $\mathrm{K}$-congruences.

Proof. Again, we may assume without loss of generality that $\vdash$ has a greatest compact theory. So, for every $\boldsymbol{A} \in \mathrm{K}$, the set $A$ is a compact $\vdash-$ filter of $\boldsymbol{A}$, whence $A \times A$ is a compact $\mathrm{K}$-congruence of $\boldsymbol{A}$ (because $\mathrm{K}$ algebraizes $\vdash$ ).

(i) $\Rightarrow$ (ii): By (i) and Theorem 4.3, the join semilattices of compact $\mathrm{K}$ congruences of all algebras in $\mathrm{K}$ are Boolean lattices. Suppose $\boldsymbol{A} \in \mathrm{K}$ is $\mathrm{K}$-subdirectly irreducible. The unique cover $\mu$ of id $A$ in the lattice of $\mathrm{K}$ congruences of $\boldsymbol{A}$ is a principal $\mathrm{K}$-congruence, whence it is compact, as is $\operatorname{id}_{A}$. Therefore, $\mu$ is also the unique atom in the semilattice of compact $\mathrm{K}$-congruences of $\boldsymbol{A}$, but a Boolean lattice with a unique atom has just two elements. So, because $A \times A$ is also compact, $\mu=A \times A$, i.e., $\boldsymbol{A}$ is $\mathrm{K}$-simple. This shows that $\mathrm{K}$ is relatively semisimple. Since EDPRC is the algebraic counterpart of the DDT [10], we can infer (ii) from Corollary 4.4.

(ii) $\Rightarrow$ (iii) follows from Lemma 5.3 and Corollary 5.2.

(iii) $\Rightarrow$ (i): If (iii) holds, then it persists for arbitrary $\boldsymbol{A}$ (as opposed to $\boldsymbol{A} \in \mathrm{K}$ ), by the remark preceding Theorem 3.10. Then (i) follows from Theorem 4.3 , because $\mathrm{K}$ algebraizes $\vdash$.

Corollary 5.5. Let $\vdash$ be a strongly algebraizable deductive system with a greatest compact theory. Then $\vdash$ has a classical inconsistency lemma iff it is algebraized by a filtral variety.

When a relatively semisimple quasivariety with EDPRC satisfies the hypotheses and equivalent conditions of Theorem 5.4, it need not be a variety. (A counter-example is given at the end of Section 6.)

Recall that a class of similar algebras is strictly elementary if it is axiomatized by a finite set of first order sentences. A deductive system is said to be finitely axiomatized if it is the deducibility relation of some formal system having only finitely many axioms and inference rules. When a quasivariety $\mathrm{K}$ algebraizes a deductive system $\vdash$, then $\mathrm{K}$ is finitely axiomatized iff $\vdash$ is. This follows from Theorems 2.17 and 4.7 in [8], and it relies on the finitarity of $\vdash$. From these observations and Theorem 5.4, we conclude:

Corollary 5.6. Let $\vdash$ be a deductive system that is algebraized by some quasivariety $\mathrm{K}$, where $\vdash$ has a classical inconsistency lemma. Then $\vdash$ is finitely axiomatized iff the class of $\mathrm{K}$-simple algebras in $\mathrm{K}$ is strictly elementary.

Proof. K is relatively semisimple with EDPRC, by Theorem 5.4. So, by Corollary 5.2 and its proof, $\mathrm{K}$ is relatively congruence distributive and the 
compact $\mathrm{K}$-congruences of algebras in $\mathrm{K}$ are closed under finite intersections. Czelakowski and Dziobiak have shown that a quasivariety $Q$ with these two properties is finitely axiomatized iff $Q_{\mathrm{FSI}}$ is strictly elementary [16, Thm. 3.4]. But $\mathrm{K}_{\mathrm{FSI}}$ is the class of $\mathrm{K}$-simple (or trivial) algebras in $\mathrm{K}$, again by the proof of Corollary 5.2.

Corollary 5.7. If two categorically equivalent varieties algebraize deductive systems $\vdash$ and $\vdash^{\prime}$, then $\vdash$ has a [classical] inconsistency lemma iff $\vdash^{\prime}$ does.

Proof. The category equivalence functor preserves the isomorphism type of the congruence lattice of any algebra, and a lattice isomorphism between complete lattices restricts to an isomorphism between their semilattices of compact elements, so the result follows from Theorems 3.10 and 5.4.

A similar result holds for the DDT.

\section{ExAmples}

The filtral variety BA of all Boolean algebras is the only nontrivial semisimple variety of Heyting algebras. Thus, by Theorem 5.4, no consistent axiomatic extension of intuitionistic propositional logic (IPL) has a classical inconsistency lemma, except for classical propositional logic (CPL). Similarly, CPL is the only axiomatic consistent extension of the $\rightarrow, \perp$ fragment of IPL having a classical inconsistency lemma. Corollaries 4.4 and 4.5 do not add up to a characterization of classical inconsistency lemmas, because IPL has a DDT and the congruence lattices of Heyting algebras are distributive and pseudo-complemented (indeed, the compact congruences form distributive lattices with 0 ).

IPL is itself an axiomatic extension of the contraction-less substructural logic $\mathbf{F} \mathbf{L}_{\text {ew }}$ of [24]. $\mathbf{F L}_{\mathbf{e w}}$ still has a greatest compact theory, generated by $\{\perp\}$. When a variety $\mathrm{K}$ algebraizes an axiomatic extension of $\mathbf{F L}_{\mathbf{e w}}$, then $\mathrm{K}$ is filtral iff it is a discriminator variety, iff the theorems of the extension include $p \vee\left(p^{k} \rightarrow \perp\right)$ for some positive integer $k$ (see [31] or [24, Chap. 11]). By Theorem 5.4, these are exactly the axiomatic extensions of $\mathbf{F L}_{\mathbf{e w}}$ with a classical inconsistency lemma. The respective classical IL-sequences are

$$
\left\{\left\{\left(v_{1} \odot \ldots \odot v_{n}\right)^{k} \rightarrow \perp\right\}: n \in \mathbb{N}\right\},
$$

where $\odot$ denotes fusion.

The relative subvarieties $\mathrm{K}$ of the quasivariety of bounded BCK-algebras algebraize the axiomatic extensions of the $\rightarrow, \perp$ fragment $\mathbf{B C K} \mathbf{K}_{\perp}$ of $\mathbf{F L}_{\mathbf{e w}} \cdot{ }^{4}$ In any such $\mathrm{K}$, the $\mathrm{K}$-simple algebras are simple in the absolute sense [26], and semisimplicity has been characterized syntactically by Torrens [39]. It

\footnotetext{
${ }^{4} \mathrm{~A}$ relative subvariety of a quasivariety $\mathrm{M}$ is the intersection of $\mathrm{M}$ with a variety of the same type.
} 
follows from [39] that an axiomatic extension of $\mathbf{B C K} \mathbf{K}_{\perp}$ has a classical inconsistency lemma iff its theorems include

$$
\left(p \rightarrow^{k+1} q\right) \rightarrow\left(p \rightarrow^{k} q\right) \text { and }(p \rightarrow q) \rightarrow\left(\left(\left(p \rightarrow^{m} \perp\right) \rightarrow q\right) \rightarrow q\right)
$$

for suitable $k, m \in \mathbb{N} .{ }^{5}$ In this case, the algebraic counterpart $\mathrm{K}$ is a discriminator variety iff $(p \rightarrow \perp) \rightarrow \perp$ and $p$ are logically equivalent in the extension of $\mathbf{B} \mathbf{C K} \mathbf{K}_{\perp}$. The proof, and an example where $\mathbf{K}$ is a (filtral) variety but not a discriminator variety, can be found in [39]. The instances in which $(p \rightarrow \perp) \rightarrow \perp$ and $p$ are equivalent include the Eukasiewicz $k$-valued $\operatorname{logics}(k \in \mathbb{N})$. The classical IL-sequence would normally be written as

$$
\left\{\left\{\neg\left(\left(v_{1} \odot \ldots \odot v_{n}\right)^{k}\right)\right\}: n \in \mathbb{N}\right\}
$$

in this context, where $p \odot q$ and $\neg(p \rightarrow \neg q)$ are interchangeable.

The infinite-valued Łukasiewicz logic has no classical inconsistency lemma, as it is algebraized by the variety of all $M V$-algebras, which lacks EDPC. In fact, this logic has no inconsistency lemma of the ordinary kind. The argument, due to the referee, is as follows. In the opposite case, it would follow from the inconsistency of $\Psi_{1}(p) \cup\{p\}$ and the local deduction theorem that $\Psi_{1}(p) \vdash \neg\left(p^{n}\right)$ for some $n \in \mathbb{N}$. Then, for each $m \in \mathbb{N}$, the inconsistency of $m p \wedge \neg p$ would entail the theoremhood of $\Psi_{1}(m p \wedge \neg p)$, and hence that of $\neg\left((m p \wedge \neg p)^{n}\right)$. But, for each $n$, we can choose an $m$ and a value of $p$ in the real interval $(0,1)$ for which $\neg\left((m p \wedge \neg p)^{n}\right)$ doesn't take the value 1 in the usual MV-algebra on $[0,1]$.

The product logic $\boldsymbol{\Pi}$ of [29] is also algebraized by a variety without EDPC, but it does have an inconsistency lemma of the ordinary kind, with

$$
\left\{\left\{\neg\left(v_{1} \odot \ldots \odot v_{n}\right)\right\}: n \in \mathbb{N}\right\}
$$

as IL-sequence. This shows that a strongly algebraizable system with an inconsistency lemma (and hence a greatest compact theory) need not have a DDT; in particular, Corollary 3.9 is not reversible. To refute EDPC in this context, consider the natural product algebra $\boldsymbol{A}$ on the ordered multiplicative submonoid

$$
\{0\} \cup\left\{2^{-n}: n \in\{0\} \cup \mathbb{N}\right\}
$$

of the reals, and let $\boldsymbol{B}=\boldsymbol{A}^{\mathbb{N}} / U$, where $U$ is a free ultrafilter over $\mathbb{N}$. For $\overline{1}, \bar{a}, \bar{b} \in A^{\mathbb{N}}$, if $\overline{1}(n)=1$ and $\bar{a}(n)=\frac{1}{2}$ and $\bar{b}(n)=2^{-n}$ for all $n$, then $\langle\bar{b} / U, \overline{1} / U\rangle \notin \mathrm{Cg}^{\boldsymbol{B}}(\bar{a} / U, \overline{1} / U)$, although $\langle\bar{b}(n), \overline{1}(n)\rangle \in \mathrm{Cg}^{\boldsymbol{A}}(\bar{a}(n), \overline{1}(n))$ for all $n$. So, by Łos' Theorem on ultraproducts, ' $\langle z, w\rangle \in \operatorname{Cg}(x, y)$ ' can't be equivalent (in product algebras) to a finite conjunction of equations in $x, y, z, w$.

The weakening axiom $p \rightarrow(q \rightarrow p)$ is a theorem of $\mathbf{F L}_{\mathbf{e w}}$. Among the substructural logics without weakening are the uninorm-based fuzzy logic IUML of [37] and its consistent extensions. These systems have connectives $\rightarrow, \wedge, \neg$ and constants $\mathbf{t}, \perp$ such that $\neg \neg p \leftrightarrow p$ and $(p \rightarrow \neg q) \rightarrow(q \rightarrow \neg p)$

\footnotetext{
${ }^{5}$ Here, $p \rightarrow{ }^{0} q:=q$ and $p \rightarrow{ }^{k+1} q:=p \rightarrow\left(p \rightarrow{ }^{k} q\right)$.
} 
are theorems. They are incomparable with the consistent extensions of $\mathbf{F L}_{\text {ew }}$, but stronger than the system $\mathbf{R} \mathbf{M}^{\mathbf{t}}$ from relevance logic. All consistent extensions of IUML are axiomatic [25], with a DDT and a greatest compact theory, generated by $\{\perp\}$. The form of the DDT is $\Gamma \cup\{\alpha\} \vdash \beta$ iff $\Gamma \vdash(\alpha \wedge \mathbf{t}) \rightarrow \beta$.

IUML is algebraized by the variety $\mathrm{OSM}_{\perp}$ of bounded odd Sugihara monoids (see $[25,35]$ and their references). It is shown in [25] that $\mathrm{OSM}_{\perp}$ is categorically equivalent to the variety of Gödel algebras - a subvariety of Heyting algebras. So, by Corollary 5.7, no consistent extension of IUML has a classical inconsistency lemma, except for the largest such extension IUML $_{3}$, whose algebraic counterpart is categorically equivalent to BA. (A variety $\mathrm{K}$ is categorically equivalent to $\mathrm{BA}$ iff it is generated by a primal algebra, see $[30,17,36]$. In this case, $\mathrm{K}$ is a discriminator variety.)

The variety that algebraizes $\mathbf{I U M L}_{3}$ is generated by a well known algebra $\boldsymbol{Z}_{3}^{\perp}$ on the idempotent commutative ordered monoid $-1<0<1$, where 0 is the identity and $-1 \odot 1=-1$. In this algebra, $\wedge$ is the minimum operation, $\neg$ is the usual additive inversion, $\mathbf{t}=0, \perp=-1$, and $x \rightarrow y:=\neg(x \odot \neg y)$. Even in $\mathbf{I U M L}_{3}$, the formulas $\neg p$ and $p \rightarrow \perp$ are not logically equivalent.

It can be verified that

$$
\left\{\left\{v_{1} \rightarrow \ldots \rightarrow v_{n} \rightarrow \perp\right\}: n \in \mathbb{N}\right\}
$$

is a classical IL-sequence for $\mathbf{I U M L}_{3}$, and also for its $\rightarrow, \perp$ fragment. The $\rightarrow$ and $\rightarrow, \perp$ fragments of $\mathbf{I U M L}_{3}$ have a common DDT, viz. $\Gamma \cup\{\alpha\} \vdash \beta$ iff $\Gamma \vdash(\alpha \rightarrow(\beta \rightarrow \beta)) \rightarrow(\alpha \rightarrow \beta)$, cf. [1]. The $\rightarrow, \perp$ fragment is algebraized by the smallest quasivariety $Q$ containing the $\rightarrow, \perp$ reduct of $\boldsymbol{Z}_{3}^{\perp}$. So, Q is relatively semisimple with EDPRC, by Theorem 5.4, but it is not a variety. Indeed, the $\rightarrow, \perp$ reduct of $\boldsymbol{Z}_{3}^{\perp}$ is Q-simple, but not simple, as it admits a factor algebra on $\{\{-1,1\},\{0\}\}$, not belonging to $\mathrm{Q}$. In particular, an algebraizable system with a classical inconsistency lemma need not be strongly algebraizable.

Acknowledgment. I am grateful to Wiesław Dziobiak for raising the question: what is the algebraic counterpart of the inconsistency lemma? I also thank an anonymous referee for correcting an error in one of the examples.

\section{REFERENCES}

[1] A. Avron, Multiplicative conjunction as an extensional conjunction, Logic J. of the IGPL 5 (1997), 181-208.

[2] G. Bergman, Sulle classi filtrali di algebre, Ann. Univ. Ferrara, Sez. VII 27 (1971), $35-42$.

[3] W.J. Blok, P. Köhler, D. Pigozzi, The algebraization of logic, manuscript, 1983.

[4] W.J. Blok, P. Köhler, D. Pigozzi, On the structure of varieties with equationally definable principal congruences II, Algebra Universalis 18 (1984), 334-379.

[5] W.J. Blok, D. Pigozzi, On the structure of varieties with equationally definable principal congruences I, Algebra Universalis 15 (1982), 195-227. 
[6] W.J. Blok, D. Pigozzi, A finite basis theorem for quasivarieties, Algebra Universalis 22 (1986), 1-13.

[7] W.J. Blok, D. Pigozzi, Protoalgebraic logics, Studia Logica 45 (1986), 337-369.

[8] W.J. Blok, D. Pigozzi, 'Algebraizable Logics', Memoirs of the American Mathematical Society 396, Amer. Math. Soc., Providence, 1989.

[9] W.J. Blok, D. Pigozzi, Algebraic semantics for universal Horn logic without equality, in A. Romanowska, J.D.H. Smith (eds.), 'Universal Algebra and Quasigroup Theory', Heldermann Verlag, Berlin, 1992, pp. 1-56.

[10] W.J. Blok, D. Pigozzi, Abstract algebraic logic and the deduction theorem, manuscript, 1997. [See http://orion.math.iastate.edu/dpigozzi/ for updated version, 2001.]

[11] W.J. Blok, J.G. Raftery, Ideals in quasivarieties of algebras, in X. Caicedo, C.H. Montenegro (eds.), 'Models, Algebras and Proofs', Lecture Notes in Pure and Applied Mathematics, Vol. 203, Marcel Dekker, New York, 1999, pp. 167-186.

[12] J. Czelakowski, Algebraic aspects of deduction theorems, Studia Logica 44 (1985), 369-387.

[13] J. Czelakowski, Local deduction theorems, Studia Logica 45 (1986), 377-391.

[14] J. Czelakowski, Relatively congruence-distributive subquasivarieties of filtral varieties, Bull. Sect. Logic 19 (1990), 66-70.

[15] J. Czelakowski, 'Protoalgebraic Logics', Kluwer, Dordrecht, 2001.

[16] J. Czelakowski, W. Dziobiak, Congruence distributive quasivarieties whose finitely subdirectly irreducible members form a universal class, Algebra Universalis 27 (1990), $128-149$

[17] B. Davey, H. Werner, Dualities and equivalences for varieties of algebras, 'Contributions to Lattice Theory' (Proc. Conf. Szeged, 1980), Coll. Math. Soc. János Bolyai, No. 33, North Holland, pp. 101-275.

[18] W. Dziobiak, Finitely generated congruence distributive quasivarieties of algebras, Fund. Math. 133 (1989), 45-57.

[19] J.M. Font, R. Jansana, 'A General Algebraic Semantics for Sentential Logics', Lecture Notes in Logic 7, Springer-Verlag, 1996.

[20] J.M. Font, R. Jansana, D. Pigozzi, A survey of abstract algebraic logic, and Update, Studia Logica 74 (2003), 13-97, and 91 (2009), 125-130.

[21] R. Franci, Filtral and ideal classes of universal algebras, Quaderni dell'Instituto di Matematica dell'Universita di Siena (1976).

[22] E. Fried, G. Grätzer, R. Quackenbush, Uniform congruence schemes, Algebra Universalis 10 (1980), 176-189.

[23] E. Fried, E.W. Kiss, Connection between the congruence lattices and polynomial properties, Algebra Universalis 17 (1983), 227-262.

[24] N. Galatos, P. Jipsen, T. Kowalski, H. Ono, 'Residuated Lattices. An Algebraic Glimpse at Substructural Logics', Studies in Logic and the Foundations of Mathematics 151, Elsevier, 2007.

[25] N. Galatos, J.G. Raftery, A category equivalence for odd Sugihara monoids and its applications, J. Pure Appl. Algebra 216 (2012), 2177-2192.

[26] J. Gispert, A. Torrens, Bounded BCK-algebras and their generated variety, Math. Logic Quarterly 53 (2007), 206-213.

[27] V. Glivenko, Sur quelques points de la logique de M. Brouwer, Bull. Acad. des Sci. de Belgique 15 (1929), 183-188.

[28] G. Grätzer, 'General Lattice Theory', Birkhäuser Verlag, Basel, 1978.

[29] P. Hájek, 'Metamathematics of Fuzzy Logic', Kluwer, Dordrecht, 1998.

[30] T.K. Hu, Stone duality for primal algebra theory, Math. Z. 110 (1969), 180-198.

[31] T. Kowalski, Semisimplicity, EDPC and discriminator varieties of residuated lattices, Studia Logica 77 (2005), 255-265.

[32] R. Magari, Varietà a quozienti filtrali, Ann. Univ. Ferrara, Sez. VII 14 (1969), 5-20. 
[33] R. Magari, The classification of idealizable varieties (Congruenze Ideali IV), J. Algebra 26 (1973), 152-165.

[34] R. Magari, Classi e Schemi Ideali (Congruenze Ideali V), Ann. S.N.S. Pisa (Classe di Scienza) 27 (1973), 687-706.

[35] E. Marchioni, G. Metcalfe, Interpolation properties for uninorm based logics, Proceedings of ISMVL 2010, IEEE Computer Society Press (2010), 205-210.

[36] R. McKenzie, An algebraic version of categorical equivalence for varieties and more general algebraic categories, in A. Ursini, P. Aglianò (eds.), 'Logic and Algebra', Lecture Notes in Pure and Applied Mathematics, Vol. 180, Marcel Dekker, New York, 1996, pp. 211-243.

[37] G. Metcalfe, F. Montagna, Substructural fuzzy logics, J. Symbolic Logic 72 (2007), 834-864.

[38] D. Pigozzi, Finite basis theorems for relatively congruence-distributive quasivarieties, Trans. Amer. Math. Soc. 310 (1988), 499-533.

[39] A. Torrens, Semisimplicity and the discriminator in bounded BCK-algebras, Algebra Universalis 63 (2010), 1-16.

[40] R. Wójcicki, 'Theory of Logical Calculi', Kluwer, Dordrecht, 1988.

Department of Mathematics and Applied Mathematics, University of Pretoria, Private Bag X20, Hatfield, Pretoria 0028, South Africa

E-mail address: james.raftery@up.ac.za 